\title{
Anesthetic experience using total intravenous anesthesia in a patient with Isaacs' syndrome -A case report-
}

\author{
Young Mi Kim, Sang Hoon Lee, Cheol Sig Han, Eun Mi Choi, Young Ryong Choi, and Mi Hwa Chung \\ Department of Anesthesiology and Pain Medicine, Kangnam Sacred Heart Hospital, Hallym University College of Medicine, Seoul, Korea
}

We report on an anesthetic experience with a 74-year-old female with Isaacs' syndrome, who underwent elective surgery for open rotator cuff repair. Isaacs' syndrome is a rare peripheral motor neuron disorder with clinical manifestations such as involuntary muscle twitching, cramps, mild weakness and increased sweating. To avoid prolonged neuromuscular blockade, the patient was observed with neuromuscular monitoring during total intravenous anesthesia with propofol, remifentanil, and atracurium. No adverse events were observed during the anesthetic management, and the patient recovered smoothly from the neuromuscular blockade. We describe the clinical characteristics of Isaacs' syndrome and its specific considerations in anesthesia. (Korean J Anesthesiol 2013; 64: 164-167)

Key Words: Atracurium, Isaacs' syndrome, TIVA.

Isaacs' syndrome is defined as continuous muscle fiber activity due to peripheral nerve hyperexcitability or neuromyotonia. Symptoms related to the disorder include cramps, muscle stiffness, pseudomyotonia and muscle twitching (visible myokymia). Hyperhidrosis, muscle weakness, muscle hypertrophy, and hyporeflexia have also been reported [1,2]. Normally, the disease has its onset in early adulthood but it can strike anyone at anytime. Causes of Isaacs' syndrome are sporadic, including inherited, hematologic malignancies, and autoimmune conditions in some patients associated with peripheral neuropathy. Diagnosis is confirmed by history, physical findings, and electromyographic (EMG) studies.
Autoantibodies directed against the voltage-gated potassium channels (VGKC) of the peripheral nerves have been demonstrated [2]. For treatment, anticonvulsive medications or immunomodulatory therapy usually controls the symptoms [3].

The ideal anesthetic for patients with Isaacs' syndrome is not known, though the major consideration is the use of neuromuscular blocking agents. The use of total intravenous anesthesia (TIVA) in Isaacs' syndrome patients had not been previously reported. Therefore, our case, in which we used TIVA in a patient with Isaacs' syndrome for open rotator cuff (RC) repair, seems to be the first report of its use in these patients.

Received: April 3, 2012. Revised: April 25, 2012. Accepted: May 3, 2012.

Corresponding author: Mi Hwa Chung, M.D., Department of Anesthesiology and Pain Medicine, Kangnam Sacred Heart Hospital, Hallym University College of Medicine, 948-1, Daerim 1-dong, Yeongdeungpo-gu, Seoul 150-950, Korea. Tel: 82-2-829-5230, Fax: 82-2-845-1571, E-mail: mhchung20@hallym.or.kr

(c) This is an open-access article distributed under the terms of the Creative Commons Attribution Non-Commercial License (http:// creativecommons.org/licenses/by-nc/3.0/), which permits unrestricted non-commercial use, distribution, and reproduction in any medium, provided the original work is properly cited. 


\section{Case Report}

A 74 year-old female patient, $158 \mathrm{~cm}$ tall and weighing $62 \mathrm{~kg}$, was diagnosed with a full thickness RC tear of her right shoulder. She was scheduled to undergo open RC repair. Her physical status was American Society of Anesthesiologists physical status II. Her prior medical history included hypertension, stable angina, and diabetes mellitus, which were well controlled with medication. Four years previously, the patient had experienced painless cramps in both hands and feet and excessive sweating without any palpitations, even during the winter season. Then, two years later she was diagnosed with Isaacs' syndrome through EMG. The nerve conduction study was unremarkable. Studies for serum anti-VGKC antibodies were not performed and antiacetylcholine-receptor antibodies produced negative results. The patient complained of gradual progressive painless flexion of her left ring and little fingers, which led to contractures (Fig. 1). The fingers could only be extended passively. No other neurologic abnormalities and sensory deficits were noted. A course of intravenous immunoglobulin was ineffective, and the patient was placed on a regimen of oral carbamazepine $200 \mathrm{mg}$ and gabapentin $300 \mathrm{mg}$ per day. She showed some improvement in symptoms without complete resolution.

Serum electrolytes including calcium, magnesium, sodium, and potassium ions were within the normal limits. Thyroid hormone levels, creatine kinase, and aldonase were also normal. Computerized tomographic scan of the thorax did not reveal thymoma. Pulmonary function tests showed a normal pattern. There was no family history of neuromuscular disease. The patient had undergone general anesthesia for spinal stenosis before her Isaacs' syndrome diagnosis in another

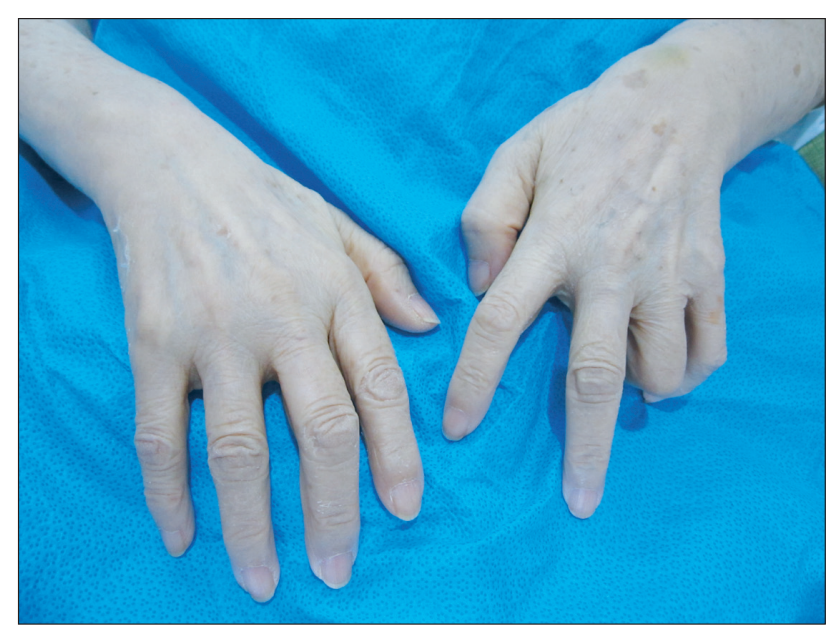

Fig. 1. Resting position of hands. There is flexion deformity in the left ring and little fingers, most marked in the ring finger. hospital. Unfortunately, the details of the anesthetic agents used were unavailable.

Upon arrival in the operating room, the patient's blood pressure was $155 / 82 \mathrm{mmHg}$, heart rate was 51 beats/min, and oxygen saturation was $97 \%$. She was premedicated with intravenous glycopyrrolate $0.2 \mathrm{mg}$ before the surgery. Preoperative monitoring including ECG, pulse oximeter, capnogram, and direct arterial pressure monitoring via the left radial arterial cannula was performed. Bispectral index (BIS) and train-of-four (TOF) monitors were attached to the patient. Three minutes after preoxygenation, lidocaine $40 \mathrm{mg}$ was administered prior to target controlled infusion (TCI) of propofol (target blood concentration $2.5 \mu \mathrm{g} / \mathrm{ml}$ ) and remifentanil (target blood concentration $2.0 \mathrm{ng} / \mathrm{ml}$ ). The patient rapidly fell into sleep with loss of eyelid reflex but abnormal muscle activity still persisted. When the numerical value of the BIS was 48 , atracurium 30 $\mathrm{mg}(0.5 \mathrm{mg} / \mathrm{kg})$ was administered. Ninety seconds later, the involuntary cramps were arrested and no response to TOF stimuli ( $2 \mathrm{~Hz}$ for $2 \mathrm{sec}$ at 10 -sec intervals) was observed. The patient was grade IIb on the modified Cormack and Lehane scoring system on laryngoscopy. Only the patient's glottis and vocal cords were visible. Nevertheless, endotracheal intubation was performed without complications. The patient was ventilated with a mixture of oxygen and air $\left(\mathrm{FiO}_{2} 0.5\right)$. Warm forced air devices were used to maintain body temperature.

During anesthesia, the patient showed no specific abnormalities of her vital signs, and an open RC repair was smoothly performed in 90 minutes. At the end of the surgery, the propofol and remifentanil infusion was stopped and the patient resumed spontaneous breathing. On the nerve stimulator, the TOF ratio was kept above 0.90 . After the patient had recovered spontaneous respiration to a sufficient extent, glycopyrrolate $0.4 \mathrm{mg}$ and pyridostigmine $15 \mathrm{mg}$ were intravenously injected to reverse the muscle relaxation. Extubation was performed at a final TOF ratio of 1 . The time from the end of surgery to extubation was about 10 minutes. We then moved the patient to the intensive care unit for continuous hemodynamic monitoring. One day after the operation, the patient was transferred to the general ward, but she complained of difficult urination. Urologic evaluation revealed a flaccid-type neurogenic bladder, and a suprapubic catheter was placed for urination. There were no further complications, and on the 22nd day after the operation, the patient was discharged from the hospital.

\section{Discussion}

Isaacs' syndrome is a disorder of peripheral nerve hyperexcitability, and usually an acquired immune-mediated disorder occurring in isolated form or in association with autoimmune diseases, such as myasthenia gravis $[1,4]$. It is caused by anti- 
bodies directed toward the slow potassium channels in terminal nerve fibers resulting in their down-regulation, which leads to repeated action potentials. A characteristic of the disease in that these continuous spontaneous muscle action potentials persist at rest and even during sleep. Stiffness without severe pain is more remarkable in the distal than proximal muscles. Hyperhidrosis may be related to increased cholinergic activity at the nerve endings in the sweat glands. Typical EMG findings show continuous single motor unit discharges occurring as doublets, triplets, or multiplet single unit discharges [2]. Supporting the diagnosis is the patient's symptomatic reaction to carbamazepine and phenytoin [3]. Since the advent of plasmapheresis and immunoglobulin therapy, the recovery time of Isaacs' syndrome patients has significantly improved, although the effectiveness of treatment diminishes if the diagnosis is delayed as in this patient. Considering the rarity of the disease, few reports have been described regarding anesthesia in patients with neuromyotonia. Anesthesiologists should be aware that there is a possible association of the condition with myasthenia gravis, thymoma, rheumatoid disease, Guillain-Barre syndrome and Lambert-Eaton syndrome [5]. Isaacs's syndrome patients require individual management in preparation for surgery, appropriate selection and administration of anesthetics, and close monitoring during surgical procedures.

The abnormal muscle activity in these patients persists during general anesthesia with propofol or thiopental but ceases during epidural anesthesia. Successful epidural analgesia for labor has been reported [6]. A review of the literature reveals that involuntary muscle contractions persist during spinal anesthesia. On the other hand, there have been isolated case reports demonstrating cessation of these contractions during spinal anesthesia [7]. The spontaneous muscle action potentials did not change under proximal nerve blockade, however, they were decreased by distal motor nerve blockade because the abnormal discharge originated from the motor nerve terminals $[1,8,9]$.

The most significant anesthetic concern in the patient discussed here was the use of neuromuscular blocking agents. A d-tubocurarine regional test showed that sensitivity had increased, with abolition of spontaneous muscular activity potential and slow recovery of muscle activity potential, similar to myasthenia gravis $[1,3]$. Neostigmine did reverse the block but the response was slow [6]. Ginsburg et al. [10] observed hypersensitivity to rocuronium in a patient with acquired neuromyotonia and a past medical history notable for prolonged postoperative paralysis. This increase in sensitivity may be explained by down-regulation of acetylcholine receptors in response to chronic high acetylcholine concentrations. In patients with neuromyotonia, neuromuscular monitoring in the form of a nerve stimulator with an accurate, objective assessment of muscle response such as acceleromyography, mechanomyography, or electromyography is necessary. Atracurium presents a good choice for neuromuscular blockade in these patients because of its relatively short duration of action and reliable pharmacokinetics. In case of our patient, it was safely used to provide optimal muscle relaxation without increasing sensitivity. In addition, the activity disappeared after intravenous injection of succinylcholine $[1,9]$, but it is advisable to avoid succinylcholine and halogenated anesthetics, which can cause a state of acute muscle denervation in such patients, possibly triggering hypercalcemic response and malignant hyperthermia.

Some therapeutic agents prescribed for cramps have drug interactions with anesthetic agents. Richard et al. [11] showed that patients chronically treated with anticonvulsants are resistant to the effect of cisatracurium and have an increased total body clearance of the drug. Additionally, Spacek et al. [12] reported that chronic anticonvulsant therapy with carbamazepine significantly shortened the rocuronium-induced neuromuscular block compared with the control patients. Knowledge of the clinical features and the interactions of common anesthetics with the anticonvulsant therapeutic agents is important in planning intraoperative management for Isaacs' syndrome patients.

It is difficult to anticipate the postoperative respiratory conditions simply based on the TOF from a single peripheral muscle. If the surgery is not disturbed, the non-relaxant technique allows early recovery of the patient to spontaneous respiration. This technique requires the use of generous local anesthetics to obtund airway reflexes during intubation. In cases where general anesthesia is indicated, it is desirable to use short-acting opioids and muscle relaxants. If the patient was induced with steroid muscle relaxants, the launch of sugammadex holds out hope to steadily reduce the incidence of postoperative residual curarization. Meanwhile, nerve blockade appears to have variable effects. An alternative is to initiate neuraxial anesthesia, depending on the operation site, with supportive non-invasive ventilation in the perioperative period. The benefits of neuraxial anesthesia include avoidance of intravenous opioids, neuromuscular blocking drugs, and anticholinesterases.

Propofol, an anesthetic agent for this patient, has benefits including hemodynamic stability, a low incidence of postoperative nausea and vomiting, and smooth emergence. After using propofol for induction and maintenance, the most noticeable features were the fast recovery, the rapid orientation, and the minimal interference with psychomotor activity and coordination after anesthesia. Concern about the possibility of intraoperative awareness could be eased by using the BIS. Some reports have also suggested that propofol sedation is safe [13]. 
Thus, an infusion of propofol supplemented with the shortacting opioid analgesic remifentanil provides more satisfactory anesthetic conditions than inhalation agents.

There have been a few reports of bulbar and laryngeal involvement in Isaacs' syndrome [4]. Jackson et al. [14] reported that a patient with Isaacs' syndrome presented with hoarseness and dyspnea caused by myokymia of the laryngeal muscles. Both weakness and continuous muscle contraction of the laryngeal muscles probably contributed to this patient's prominent respiratory problems. When the pharyngeal and laryngeal muscles are involved, the risks of pulmonary aspiration and upper airway obstruction during general anesthesia increase. In addition, the tongue and jaw become stiff, resulting in dysphagia, which can lead to difficult intubation. Respiratory function tests may be valuable in determining the degree of respiratory involvement and the likely requirement for postoperative ventilation.

Isaacs's syndrome is a rare peripheral motor neuron disorder. Its involuntary muscle activity does not change under general anesthesia and is stopped by neuromuscular blockade. In our case, the time of onset and recovery of atracurium remained in the normal range, so the patient could be extubated shortly after the operation and did not develop prolonged muscle weakness. This could be done thanks to neuromuscular monitoring by TOF and the use of TCI with propofol and remifentanil. It is advisable to perform careful neuromuscular monitoring during anesthesia to protect all patients with Isaacs' syndrome.

\section{References}

1. Isaacs H. A syndrome of continuous muscle-fibre activity. J Neurol Neurosurg Psychiatry 1961; 24: 319-25.

2. Maddison P. Neuromyotonia. Clin Neurophysiol 2006; 117: 2118-27.
3. Sinha S, Newsom-Davis J, Mills K, Byrne N, Lang B, Vincent A. Autoimmune aetiology for acquired neuromyotonia (Isaacs' syndrome). Lancet 1991; 338: 75-7.

4. Lahrmann H, Albrecht G, Drlicek M, Oberndorfer S, Urbanits S, Wanschitz J, et al. Acquired neuromyotonia and peripheral neuropathy in a patient with Hodgkin's disease. Muscle Nerve 2001; 24: 834-8.

5. Newsom-Davis J, Mills KR. Immunological associations of acquired neuromyotonia (Isaacs' syndrome). Report of five cases and literature review. Brain 1993; 116: 453-69.

6. Morgan PJ. Peripartum management of a patient with Isaacs' syndrome. Can J Anaesth 1997; 44: 1174-7.

7. Ashizawa T, Butler IJ, Harati Y, Roongta SM. A dominantly inherited syndrome with continuous motor neuron discharges. Ann Neurol 1983; 13: 285-90.

8. Sakai T, Hosokawa S, Shibasaki H, Goto I, Kuroiwa Y, Sonoda H, et al. Syndrome of continuous muscle-fiber activity: increased CSF GABA and effect of dantrolene. Neurology 1983; 33: 495-8.

9. Wallis WE, Van Poznak A, Plum F. Generalized muscular stiffness, fasciculations, and myokymia of peripheral nerve origin. Arch Neurol 1970; 22: 430-9.

10. Ginsburg G, Forde R, Martyn JA, Eikermann M. Increased sensitivity to a nondepolarizing muscle relaxant in a patient with acquired neuromyotonia. Muscle Nerve 2009; 40: 139-42.

11. Richard A, Girard F, Girard DC, Boudreault D, Chouinard P, Moumdjian R, et al. Cistaracurium-induced neuromuscular blockade is affected by chronic phenytoin or carbamazepine treatment in neurosurgical patients. Anesth Analg 2005; 100: 538-44.

12. Spacek A, Neiger FX, Krenn CG, Hoerauf K, Kress HG. Rocuroniuminduced neuromuscular block is affected by chronic carbamazepine therapy. Anesthesiology 1999; 90: 109-12.

13. Shyr MH, Ho AC, Lin CC, Hsu KY, Yang CH, Chen CH. Propofol anesthesia in a patient with Isaacs syndrome-report of a case and literature review. Acta Anaesthesiol Sin 1997; 35: 241-5.

14. Jackson DL, Satya-Murti S, Davis L, Drachman DB. Isaacs syndrome with laryngeal involvement: an unusual presentation of myokymia. Neurology 1979; 29: 1612-5. 